\title{
Rabies and the Heart
}

\author{
Bryce Alexander ${ }^{\mathrm{a}}$, Jose Patricio Lopez-Lopez ${ }^{\mathrm{b}}$, Clara Saldarriaga ${ }^{\mathrm{c}}$, Carlos I. Ponte-Negretti ${ }^{\mathrm{d}}$, \\ Ricardo Lopez-Santie ${ }^{\mathrm{e}}$, Gonzalo Emanuel Perez, Mildren del Sueldog, \\ Fernando Lanas ${ }^{\mathrm{h}}$, Kiera Liblik $^{\mathrm{a}}$, Adrian Baranchuk ${ }^{\mathrm{a}}$,
}

\begin{abstract}
As one of the neglected diseases, rabies is as a highly fatal viral infection, most prevalent in low- and middle-income regions, which produces a substantial health and economic burden. It mainly affects the central nervous system causing encephalitis, however extraneuronal involvement has been documented. Cardiac structures may be involved and can play a role in the severity of the disease. Most of the existing literature comes from case reports and case series where cardiac involvement results in myocarditis and cardiac arrhythmias. As part of the "Neglected Tropical Diseases and Other Infectious Diseases Involving the Heart" (NET-HEART Project), the objective of this article is to review all the information available on the cardiac involvement of this disease.
\end{abstract}

Keywords: Rabies; Heart; Myocarditis; Pericarditis; Cardiac arrhythmias; Blood vessels

\section{Introduction}

Rabies is a neglected tropical disease caused by the rabies virus, a single-stranded ribonucleic acid (RNA) virus belonging

Manuscript submitted January 4, 2021, accepted January 11, 2021

Published online January 24, 2021

aDivision of Cardiology, Kingston Health Science Center, Queen's University, Kingston, ON, Canada

${ }^{b}$ Centro Integral para la Prevencion de Enfermedades Cardiometabolicas (CIPCA) and Instituto de Investigaciones Masira, Universidad de Santander (UDES), Bucaramanga, Colombia

cDepartment of Cardiology and Heart Failure Clinic, Clinica Cardiovascular Santa Maria, Universidad of Antioquia, Medellin, Colombia

${ }^{\mathrm{d} C}$ ardiometabolic Medicine Unit, Instituto Medico La Floresta, Caracas, Venezuela

eDivision of Cardiology, Hospital Italiano de La Plata, Buenos Aires, Argentina

fDivision of Cardiology, Clinica Olivos, Buenos Aires, Argentina

gUniversity of Antioquia, Clinica CardioVID, Medellin, Colombia

hUniversidad de La Frontera, Temuco, Chile

${ }^{i}$ Corresponding Author: Adrian Baranchuk, Medicine and Physiology, Cardiac Electrophysiology and Pacing, Kingston General Hospital, Queen's University, ON K7L 2V7, Canada. Email: barancha@kgh.kari.net

doi: https://doi.org/10.14740/cr1216 to the Lyssavirus genus. Due to its predominant neurotropism, rabies' main clinical effect is severe encephalitis; however, involvement of other organs such as the eyes, salivary glands, and the skin have been reported $[1,2]$. Limited reports have demonstrated that the virus may have a particular tropism for the heart conduction system, leading to a range of cardiovascular complications.

\section{Epidemiology}

Human rabies is estimated to produce loss of approximately 3.7 million disability-adjusted life years (DALYs) and to produce economic losses of around $\$ 8.6$ billion (95\% confidence intervals (CIs): $2.9-21.5$ billion) annually. The most substantial economic burden is caused by premature death, followed by direct costs of post-exposure prophylaxis [3]. As a neglected tropical disease, more than $80 \%$ of deaths occur in rural areas of resource-poor regions, where the presence of non-immunized dogs and the unavailability of post-exposure prophylaxis coexist. Although the prompt administration of immunization can prevent progression of the disease, there are no effective measures to improve its prognosis once clinically established, leading to a mortality rate close to $100 \%[4,5]$. In most parts of the world, the principal hosts of rabies virus are mesocarnivores such as dogs, foxes, raccoon dogs, and raccoons. Canine rabies is responsible for $99 \%$ of human rabies cases. The canine rabies virus is endemic in most countries of Asia and Africa; however in Western Europe, North America, Japan, and some Latin American countries, it has been eliminated mainly by the massive vaccination of dogs (Fig. 1) [6]. Globally, it is estimated that human rabies causes approximately 59,000 (95\% CI: 25 - 159.00) deaths per year, attributable to its fatality rate close to $100 \%[2,5]$. However, these numbers could be even higher because of the scarce epidemiologic surveillance in low and middle-income countries. Underfunded health care systems with limited diagnostic capacity and inefficient medical and veterinary infrastructure potentially entail less case reporting. For example, in countries like India or Pakistan, human rabies is not a mandatory epidemiological notification [7]. Likely due its extremely high fatality rate there is little literature about cardiac involvement caused by the rabies virus, and most publications consist of case reports or case series reporting mainly myocarditis or ventricular and supraventricular arrhythmias.

The "Neglected Tropical Diseases and other Infectious Diseases Involving the Heart" (the NET-Heart Project) is an 

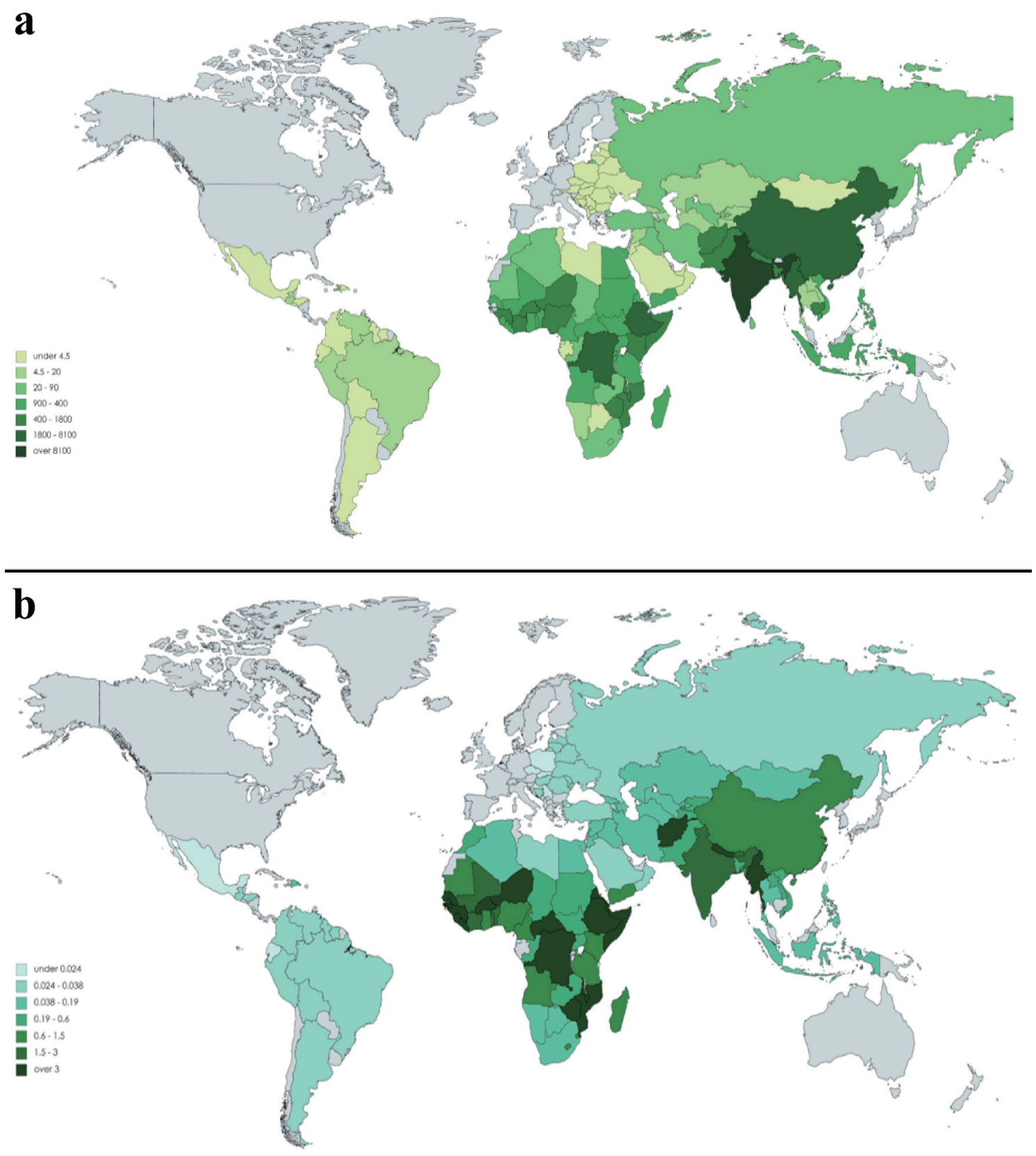

Figure 1. (a) Human deaths from rabies. (b) Death rates per capita (per 100,000 population); countries shaded in grey are free from canine rabies (Adapted from: TRS 3rd report, 2017, http://www.who.int/rabies/epidemiology/en/)

initiative of the "Emerging Leaders" section of the Interamerican Society of Cardiology which aims to expand the knowledge of the cardiovascular (CV) complications in these type of diseases, to help to identify barriers and to look for possible solutions [8-12].

\section{Methods}

We conducted a systematic review of the literature following the design and rationale of the NET- Heart Project [8]. Initial- ly, the databases from PubMed, EMBASE, and LILACS were screened to identify articles containing the MESH terms "rabies", "heart", "pericarditis", "arrhythmias", "blood vessels" and "myocarditis" limited to human studies and restricted to English. There was no restriction of a year of publication. The initial selection was made according to the inclusion criteria: randomized clinical trials, case series, observational studies, systematic reviews, and case reports. Off-topic articles were excluded. Two authors (BA and JPL) reviewed all articles' titles and abstracts in a blinded manner. After applying inclusion and exclusion criteria, selecting the potential candidates 


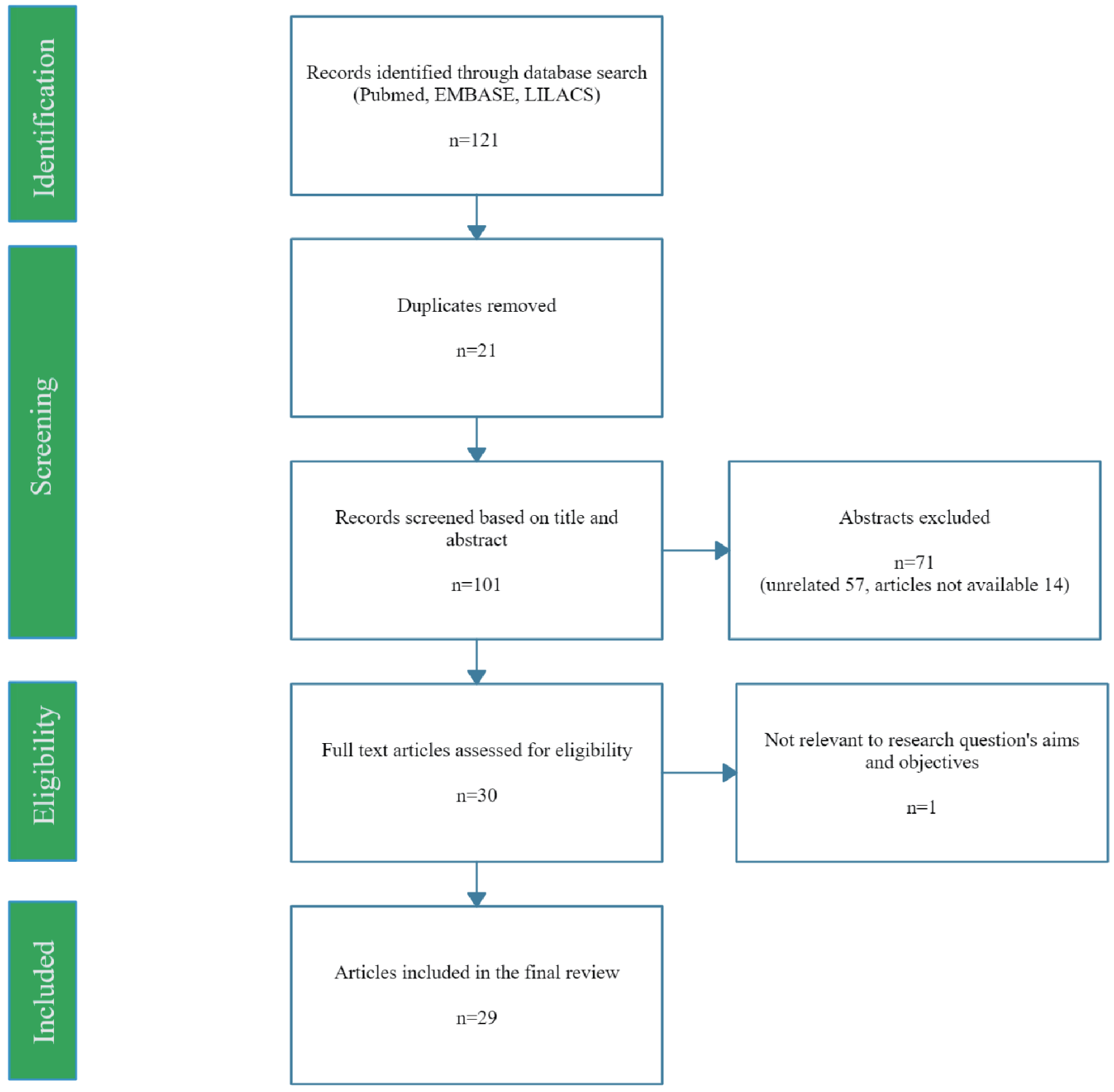

Figure 2. PRISMA flow diagram demonstrating search strategy used. PRISMA: Preferred Reporting Items for Systematic Reviews and Meta-Analyses.

with a Cohen's Kappa coefficient of 0.51, and resolving the common agreement's discrepancies, 29 articles were included were detected to be included in the review: 11 case reports, six case series, eight non-systematic reviews, three cohort studies, and one uncontrolled clinical trial. From the review of the list of references, 11 additional articles of interest were obtained (PRISMA flowchart, see Figure 2).

\section{Physiopathology and Clinical Presentation of Rabies}

The rabies virus enters through wounds caused by an infected animal or by direct contact of the animal's saliva with a human's oral or eye mucosa. After that, the virus replicates in the muscular tissue, and the virions are retrogradely transported in vesicles by rapid transport through peripheral nerve motor axons before penetrating the central nervous system (CNS) and developing into encephalitis (Fig. 3). The typical clinical stages of rabies are characterized by an incubation period that usually lasts between 1 and 2 months after contact with the infected animal followed by a prodrome lasting 1 to 10 days. During the prodromal phase the patient may exhibit non-specific symptoms, including fatigue, loss of appetite, headache, insomnia, anxiety, irritability, myalgias, visual or olfactory hallucinations, and fever. Often a progressive and intense local reaction that begins at the bite site and gradually expands and involves the limb in a non-root pattern, or the face on the ipsilateral side occurs during the early phases of the disease. The prodromal phase is followed by an acute neurologic phase which can man- 


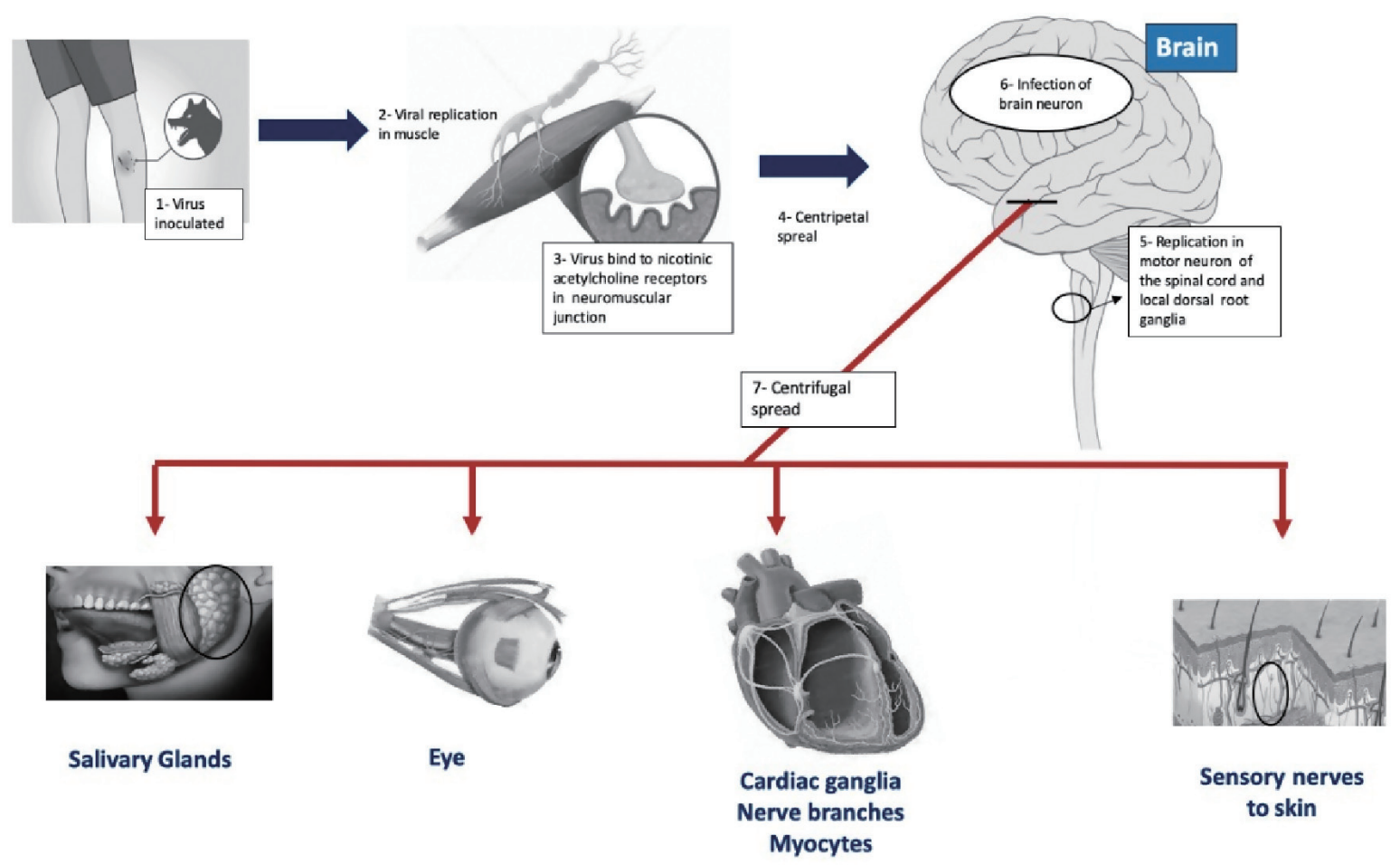

Figure 3. Mechanism of rabies infection.

ifest as encephalic rabies (80\%) or paralytic rabies $(20 \%)$.

\section{Diagnosis of Rabies}

Rabies can be diagnosed by laboratory studies or post-mortem on autopsy (Table 1). Resource limitations in health systems where human rabies virus is endemic result in the diagnosis often being made based purely on clinical findings with no laboratory testing carried out to confirm the infection. Diagnostic tests that can be used to confirm rabies virus infection antemortem in humans involve one, or a combination of: 1) viral antigen detection; 2) detection of neutralizing antibodies to the rabies virus; 3) viral RNA detection or 4) virus isolation [13].

\section{Cardiac Involvement in Rabies}

\section{Ante-mortem clinical evidence for cardiac involvement in rabies}

Cardiac involvement in rabies was first described in 1962 in a patient who developed non-specific electrocardiographic abnormalities of the ST-T segment before dying from refractory cardiovascular collapse [14]. A broad range of rabies cardiovascular clinical presentations has been described and are outlined in Table 2 [15-25]. Due to the predilection rabies virus for myocardial cells and the conduction system, cardiovascular symptoms originate from the development of myocarditis and arrhythmias. Park et al reported the first case of myocarditis presenting with an ST- elevation acute coronary syndrome. The patient presented with shortness of breath and upper limb paresthesias; the electrogram showed persistent ST-segment elevation, autonomic instability with Mobitz type II atrioventricular block, asystole, and a forthright elevation of cardiac biomarkers [15]. Typical symptoms of myocarditis such as chest pain, hypotension, and signs of heart failure have been reported [14-18, 26]. Boukas et al reported a case where an echocardiogram showed progressive dilatation, increased thickness, and irregularities of the coronary arteries suggesting a vasculitis process [27]. Arrhythmias are thought to be triggered by conduction system involvement, but the disease's oxygenation disturbances also potentiate their onset [19]. Several types of arrhythmias have been reported in rabies and are shown in Table 2 [15-25]. When cardiac involvement is present, electrocardiogram findings may mimic an acute coronary syndrome, including complete left bundle branch block, diffuse ST-segment depression, T-wave inversion, or even ST-segment elevation $[15,18,25]$. The most frequent electrocardiographic finding is sinus tachycardia, but atrial flutter, persistent atrial premature and ventricular complexes, and complete heart block have also been described [16, 20, 21].

\section{Post-mortem pathology evidence for cardiac involvement in rabies}

In post-mortem studies, the rabies virus antigen has been detected in different human tissues, including the heart $[22,23$, 28]. Cardiac involvement begins when the rabies virus enters the myocardial cells and affects the conduction system through centrifugal dissemination, being detectable in the car- 
Table 1. Diagnostic Tests for Rabies

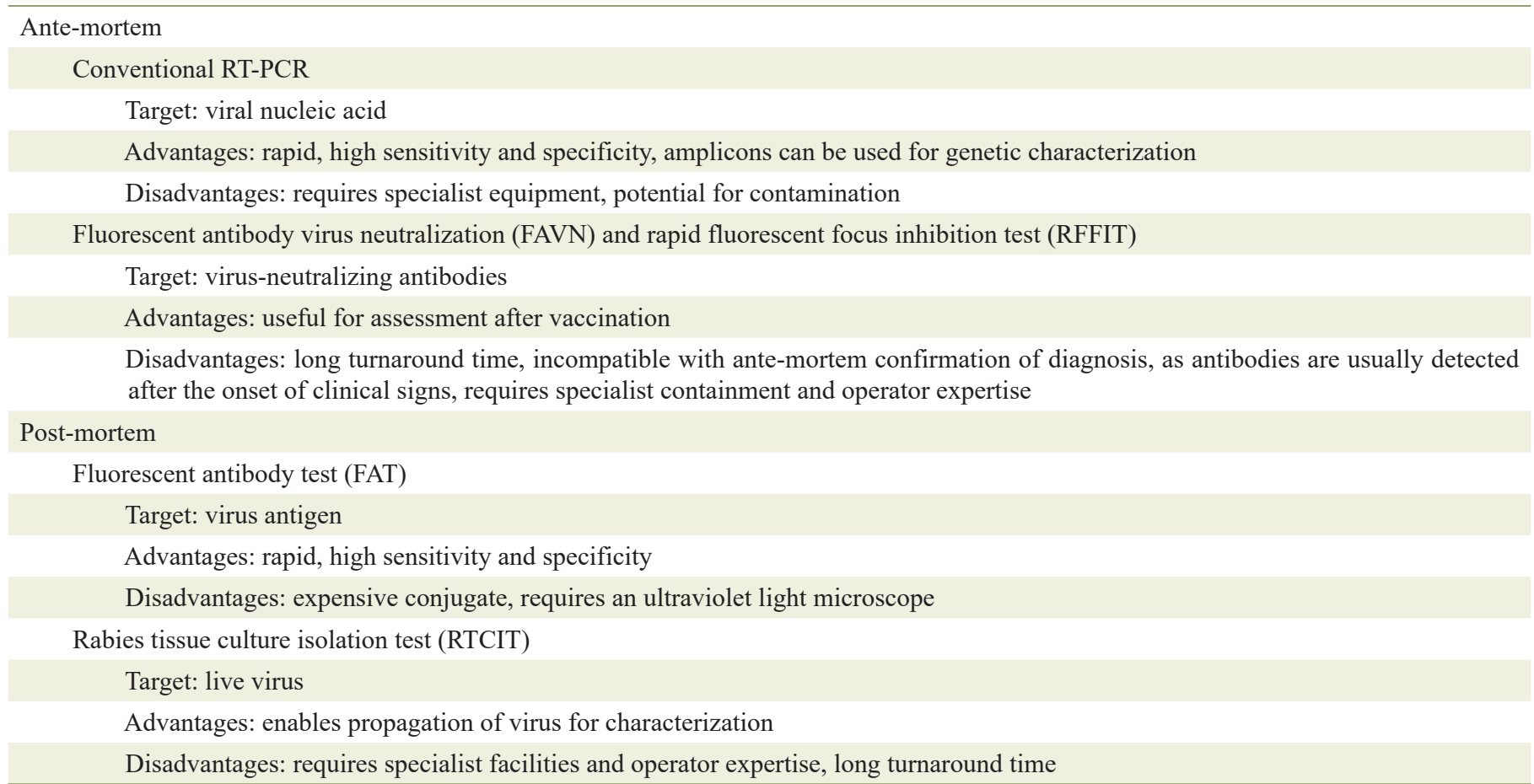

diac ganglia on the fifth day of the disease. The incidence of histopathological lesions is variable since a cardiac biopsy is not often performed to confirm cardiovascular disease [24]. Of the few studies that have been conducted, myocarditis and the presence of viral inclusion bodies in the myocardium have been described in patients who died from human rabies [17, $18,27,28]$. Duenas et al evaluated 17 patients with rabies who underwent a myocardial biopsy, and the virus was isolated in 10 patients $(58 \%)$. Of those, two revealed only focal lymphocytic infiltration limited to the interstitium, with the remaining subjects not demonstrating any significant pathological lesions [25]. Araujo et al confirmed focal interstitial myocarditis in $43.5 \%$ of 23 human rabies cases [22]. Additional viral involvement of the conduction system has been described [14, 24].

\section{Treatment}

As mentioned above, most cases of rabies occur in poverty and rural contexts. Initial management often takes place in prima-

Table 2. Summary of Studies Mentioning Cardiac Complications of Rabies

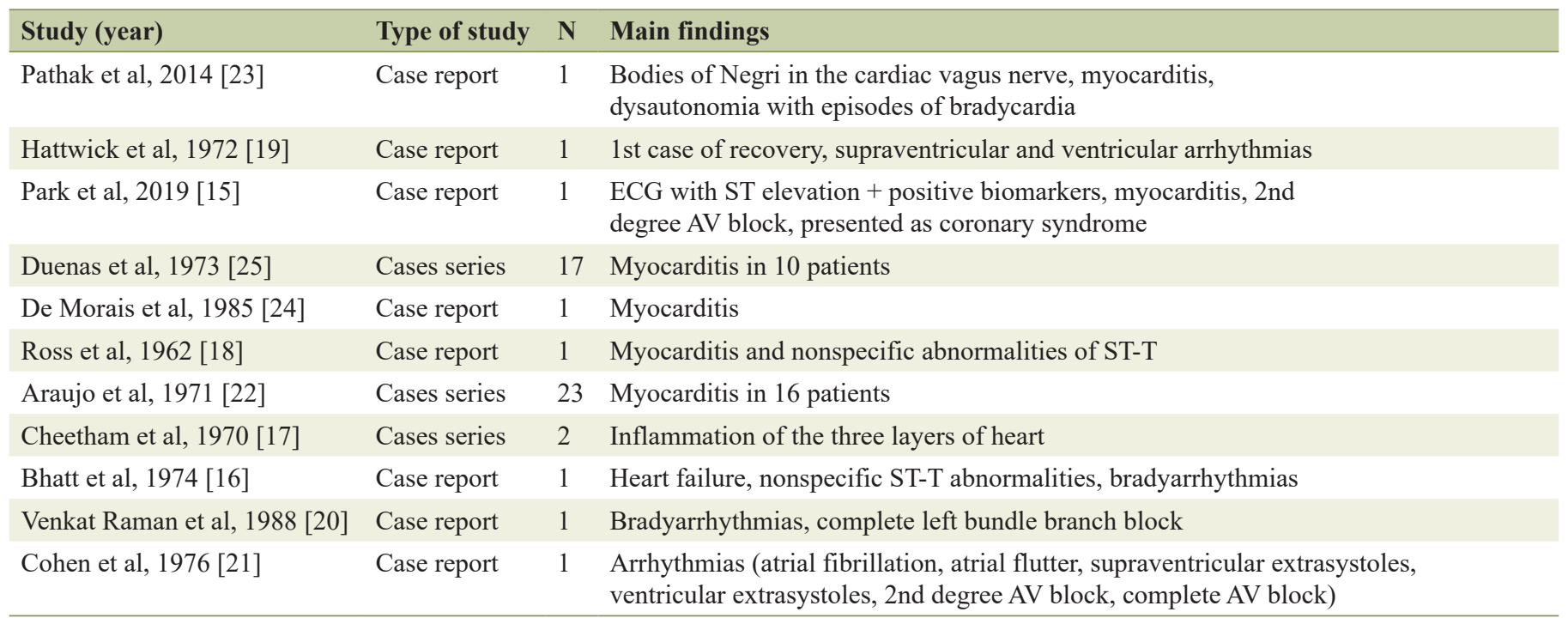


ry care centers where limited resources for basic wound-care and post-exposure prophylaxis (PEP) are common [4]. Rabies treatment revolves around adequate wound care, PEP that includes passive and active immunization, and supportive treatment in an overt case of rabies. The exposure risk assessment and the decision to administer PEP should be based on World Health Organization (WHO) guidelines [2]. PEP may include administration of the rabies vaccine and/or administration of immunoglobulins derived from hyperimmunized human or horse plasma. Once clinical rabies is established administration of rabies immune globulin is no longer recommended as these immunoglobulins do not cross the blood-brain barrier. It is important to clarify that there is no effective curative treatment against rabies once clinical signs have appeared. Most patients will die, so the treatment should have a palliative approach to administer deep sedation and avoid intubation or life-support measures. A combination of sedation and analgesia by opioids and benzodiazepines is useful to treat agitation and muscle spasms, while haloperidol is recommended to treat restlessness, agitation, hallucinations, and aggressiveness. In the case of excessive salivation, anticholinergics such as scopolamine are useful. The treatment of rabies' cardiovascular complications does not differ from its standard of care; generally, the treatment is supportive. If the most important challenge for the clinician is treating circulatory dysfunction, then vasoactive agents are the first line of treatment. In the case of suspicion of myocarditis, supportive treatment should be carried out, and when heart failure symptoms or deterioration of the ejection fraction occurs, patients should receive the standard of care. In the presence of arrhythmias, conventional antiarrhythmics such as amiodarone may be started. In the case of bradyarrhythmias, treatment with atropine and/or isoproterenol has been described, as well as placement of a temporary pacemaker.

\section{Conclusions}

Rabies continues to be a neglected disease and a public health challenge in low and middle-income countries where public health policies, poor surveillance, underreporting, misdiagnosis, and lack of coordination among all sectors involved, lead to an underestimation of the disease burden. There is evidence from both ante-mortem clinical and post-mortem pathological sources that rabies can affect the heart, where it can cause myocarditis, arrhythmias or less frequently conduction disorders (heart block); however this evidence comes primarily from case reports and case series. As the mortality rate of clinically established rabies is virtually $100 \%$, cardiac involvement does not substantially affect the trajectory of the disease. Treatment for cardiac complications of rabies does not differ from treatment of those without the disease and care is primarily supportive in nature.

\section{Acknowledgments}

This article is on behalf of the Neglected Tropical Diseases and
Other Infectious Diseases Affecting the Heart (NET-Heart) project.

\section{Financial Disclosure}

None to declare.

\section{Conflict of Interest}

The authors have no conflicts of interest to disclose.

\section{Author Contributions}

All authors made substantial contributions to design of the work. The manuscript was drafted by BA and JPL. All authors were involved in critically revising the work for important intellectual content. All authors were involved in the final approval of the version to be published.

\section{Data Availability}

Any inquiries regarding supporting data availability of this study should be directed to the corresponding author.

\section{References}

1. Jackson AC. Human Rabies: a 2016 Update. Curr Infect Dis Rep. 2016;18(11):38.

2. World Health Organization Expert Consultation on Rabies. 2018.

3. Hampson K, Coudeville L, Lembo T, Sambo M, Kieffer A, Attlan M, Barrat J, et al. Estimating the global burden of endemic canine rabies. PLoS Negl Trop Dis. 2015;9(4):e0003709.

4. Wilde H, Lumlertdacha B, Meslin FX, Ghai S, Hemachudha T. Worldwide rabies deaths prevention-A focus on the current inadequacies in postexposure prophylaxis of animal bite victims. Vaccine. 2016;34(2):187-189.

5. Willoughby RE, Jr., Tieves KS, Hoffman GM, Ghanayem NS, Amlie-Lefond CM, Schwabe MJ, Chusid MJ, et al. Survival after treatment of rabies with induction of coma. N Engl J Med. 2005;352(24):2508-2514.

6. World Health Organization. Rabies: epidemiology and burden of disease. 2017. Accessed July 5, 2020. http:// www.who.int/rabies/epidemiology/en/.

7. Banyard AC, Horton DL, Freuling C, Muller T, Fooks AR. Control and prevention of canine rabies: the need for building laboratory-based surveillance capacity. Antiviral Res. 2013;98(3):357-364.

8. Burgos LM, Farina J, Liendro MC, Saldarriaga C, Liprandi AS, Wyss F, Mendoza I, et al. Neglected tropical diseases and other infectious diseases affecting the heart. the Net-Heart project: rationale and design. Glob Heart. 
2020;15(1):60.

9. Ortiz HIA, Farina JM, Saldarriaga C, Mendoza I, Sosa Liprandi A, Wyss F, Burgos LM, et al. Human African trypanosomiasis \& heart. Expert Rev Cardiovasc Ther. 2020;18(12):859-865.

10. Gupta S, Gazendam N, Farina J, et al. Malaria and heart. J Am Coll Cardiol. 2020; In Press.

11. Scatularo CE, Ballesteros OA, Saldarriaga C, Mendoza I, Wyss F, Liprandi AS, Munera A, et al. Zika \& heart: A systematic review. Trends Cardiovasc Med. 2020.

12. Zhou Z, Ortiz Lopez HIA, Perez GE, Burgos LM, Farina JM, Saldarriaga C, Lopez-Santi R, et al. Toxoplasmosis and the Heart. Curr Probl Cardiol. 2020:100741.

13. WHO Expert Consultation on Rabies. Vol 931. 2005.

14. Metze K, Feiden W. Rabies virus ribonucleoprotein in the heart. N Engl J Med. 1991;324(25):1814-1815.

15. Park SC, Crane IM, Pal K, Cagnina RE. Rabies Encephalitis With Myocarditis Mimicking ST-Elevation Myocardial Infarction. Open Forum Infect Dis. 2019;6(6):ofz260.

16. Bhatt DR, Hattwick MA, Gerdsen R, Emmons RW, Johnson HN. Human rabies. Diagnosis, complications, and management. Am J Dis Child. 1974;127(6):862-869.

17. Cheetham HD, Hart J, Coghill NF, Fox B. Rabies with myocarditis. Two cases in England. Lancet. 1970;1(7653):921-922.

18. Ross E, Armentrout SA. Myocarditis associated with rabies. Report of a case. N Engl J Med. 1962;266:1087-1089.

19. Hattwick MA, Weis TT, Stechschulte CJ, Baer GM, Gregg MB. Recovery from rabies. A case report. Ann Intern Med. 1972;76(6):931-942.
20. Raman GV, Prosser A, Spreadbury PL, Cockcroft PM, Okubadejo OA. Rabies presenting with myocarditis and encephalitis. J Infect. 1988;17(2):155-158.

21. Cohen SL, Gardner S, Lanyi C, McDonald JR, Ree H, Southorn PA, Woodruff AW. A case of rabies in man: some problems in diagnosis and management. Br Med J. 1976;1(6017):1041-1042.

22. Araujo Mde F, de Brito T, Machado CG. Myocarditis in human rabies. Rev Inst Med Trop Sao Paulo. 1971;13(2):99-102.

23. Pathak S, Horton DL, Lucas S, Brown D, Quaderi S, Polhill S, Walker D, et al. Diagnosis, management and postmortem findings of a human case of rabies imported into the United Kingdom from India: a case report. Virol J. 2014;11:63.

24. de Morais CF, de Assis RV. Cardiac involvement in human rabies. Case report. Rev Inst Med Trop Sao Paulo. 1985;27(3):145-149.

25. Duenas A, Belsey MA, Escobar J, Medina P, Sanmartin D. Isolation of rabies virus outside the human central nervous system. J Infect Dis. 1973;127(6):702-704.

26. Liu Y, Yang X, Yang X. Rabies in coronary care unit: a case report. Biomed Res. 2018;29(8).

27. Boukas I, Dahdah N, Robitaille Y, Fournier A. Coronary artery dilatation and vasculitis in a case of rabies: similarity with Kawasaki disease? Pediatr Int. 2013;55(2):237240.

28. Jogai S, Radotra BD, Banerjee AK. Rabies viral antigen in extracranial organs: a post-mortem study. Neuropathol Appl Neurobiol. 2002;28(4):334-338. 